\title{
Overview of Analyzing Real Time Segmentation of High Definition Video using Gaussian Mixture Model Algorithm on FPGA
}

\author{
Manu K S ${ }^{1}$, Dr. Rekha K.R ${ }^{2}$, Dr. Nataraj K. R. ${ }^{3}$ \\ ${ }^{1}$ Research Scholar SSAHE, Tumkur, Karnataka, India \\ ${ }^{2,3}$ Department of ECE, SJB Institute of Technology, Bangalore, Karnataka, India
}

\begin{abstract}
In many image and video processing systems, background identification is basic and important features to perform the operations. In this paper we are analyzing different approaches, models and algorithms of segmentation in real time for background identification. We are approaching the segmentation for high definition vedio using Gaussian mixture model (GMM) and implement the same on high speed FPGA board. We are trying to improve the process at High resolution (HD) Frames per second (fps).we are targeting to achieve less hardware consumption with high resolution.
\end{abstract}

Keywords: GMM, BI, Segmentation, Vedio, FPGA, HD

\section{Introduction}

Vedio processing system is a special case of signal processing system and used in most of the electronic gadgets includes TV, DVD, Vedio players, vedio codec ${ }^{c e}$ and used for digital zooming, brightness, contrast, sharpness adjustments, color space conversion, frame rate conversion, resolutions adjustment, motion compensation, noise reduction, edge enhancement, object detection, segmentation, object tracking and many more.

The applications of vedio processing system in real time includes vedio database, security monitoring, reality interfaces, Vedio compression on live screen and many more [1].

Extracting the moving objects from the video or frame sequences is most crucial part in the video applications like surveillance, human detection, human machine interface (HMI) and traffic monitoring [2]. In video surveillance systems, background maintenance is one of the major models for the background subtraction problems. The background maintenance is used to avoid the problems in moving objects, waking persons, moving persons, shadows, foreground aperture, bootstrapping, time of the day, waving tree, camouflage, light switches and etc [4].

This paper is explained in different topics, Section 2 gives a detailed review of the different approaches for Gaussian mixture model (GMM) algorithm in background identification model used. Section 3 presents the working analysis of Gaussian mixture model (GMM) algorithm with background identification model. The conclusion of the work is presented in section 4 .

\section{Related Work}

The real time tracking system called "Pfinder" is used to track the human body and his movements. In [1], Pfinder model is best approach to find interpretation of human form with less hardware cost. Initially they are approached with single scene and single person images. Pfinder is process of tracking the objects or persons in real time and first it detect and modeling the person and then the scenes and analysis the loop using Gaussian matrix and segmentation. This models supports higher-order vision techniques in real time applications.

In paper [2][12], they propose an algorithm related to detecting the moving objects or abandoned objects from static background picture includes shadows and shading using color frames or images. They developed an efficient algorithm called background subtraction algorithm and it is able to find the local and global illumination changes includes high lights and shadows. They are used color image for decomposing the brightness and chromaticity measurement according to the scenes. They have done pixel calculation using Background subtraction algorithm, then automatic threshold calculation and clustering detection and elimination. They achieved the robustness, accuracy and amount of speeding process.

A realistic method is introduced in [3][6][11][15-16] for background subtraction. They are considering modeling for each pixel in images or frames or videos as a separate Gaussian mixture model. They are used two parameters to make model should be in robust nature. That is $\alpha=$ learning constant and $\mathrm{T}=$ Propagation of data by background. These model supports without altering the parameters monitoring indoor and outdoor scenes and human computer interface. It also supports and adapts the values of Gaussian in many modeling.

In paper [4], they introduce new system to avoid background subtraction disadvantages in video surveillance systems called "Wallflower" and it avoids the most of the critical problems.

Wallflower contains 3 processing component models like pixel level and region level and frame level modeling. In the pixel modeling, only basic classification of foreground and 


\section{International Journal of Science and Research (IJSR) \\ ISSN (Online): 2319-7064 \\ Index Copernicus Value (2013): 6.14 | Impact Factor (2014): 5.611}

background and also adapts to change the background. In region level model processing of inter-pixel calculations and also helps to redefine the pixel level process. In frame level modeling, finds the problem in light switch. If any large changes happen in the image, this will replace the other background for maintenance. In paper [5], they used background registration technique to find moving objects. And it is also similar of previous [2] [4] type except in postprocessing, they are smoothing the images and reducing much shadow effect than other techniques.

The background detection and shadow, moving object detection are presented in gray scale vedio sequences in [7][9]. The model is also same as previous techniques to get only the correct moving objects.

The FPGA Based Gaussian mixture model as a classifier is firstly designed on [8] using DA (Distributive Arithmetic).To reduce the hardware complexity using DA, they used exponential calculated circuit based on piecewise approximation. They are achieved good operating speed with less complexity and successfully implemented on FPGA.

The supreme approach for parameter estimation of FMM (Finite Mixture Model) is expectation-maximization (EM) algorithm. The drawback of FMM is components are not advanced and it is always problem for EM algorithms. So they gone with modified penalize minimum matching distance calculation for GMM based designs in [10][13][19][22] .

In [14], they use GMM as a data stream clustering and arbitrary clustering graphics. But two major problems for clustering technologies number of cluster selection and partitioning overlapping clustering. To solve this problem they go with GMM with genetic algorithm. It supports split and merge operations. So we can get accurate information.

The GMM Based background modeling and motion detection is presented in [17] and it is same previous methods and extra is motion detection is implementation on FPGA. The design is optimized and with logic resources.

For speech reorganization is design and developed with help of both hardware and software with minimum resources. using Mel Frequency Cepstral Coefficients (MFCCs) technique we can extract The speech signal features . GMM Model is used for observe the Voice information.HMM model is used as a toanalyse the statistical modeling voice information of GMM.The accuracy of recognition words are fluent and the full design is implemented on System on Programmable Chip (SoCP) .

The real time background identification for higher resolution $1080 p$ video sequences is implemented on Viterx-5 FPGA.The hardware implementation is OPENCV version of GMM.The ROM compressors and truncated multipliers are used in GMM model to reduce the hardware complexity.

\section{Gaussian Mixture Model}

In paper [3] [11][18][23] the Gaussian mixture model has been proposed and using statistical model they are generated multi modal Bg with mixture of Gaussian distributions. The Gaussian mixture model mainly contains three models includes statistical model, parameters updating and Background identification model.

\section{A. Statistical Model}

In statistical model [23], we will capture the video sequence using CMOS Camera and the model for video sequence of each pixel is made by mixture of , $\mathrm{K}^{\mathrm{ee}}$ Gaussian distributions. Each „, $\mathrm{K}^{\mathrm{e}}$ Gaussian distribution is represented by four parameters of the mixture model. It includes Weight $(w)$, Matchsum, mean $(\mu)$ and variance $\left(\sigma^{2}\right)$. The video sequence of each frame will be changed by Gaussian parameters and it"s Gaussian of each pixel. So three indices are defined based on Gaussian parameters like p, k and t. where " $p$ " is pixel index, " $\mathrm{k}$ " is Gaussian index and " $\mathrm{t}$ " is frame index.

\section{B. Parameters Update}

Fitness $\left(F_{k, t}\right)$ is a sorted model in descending order of a parameter in each pixel for the $\mathrm{K}$ Gaussian distribution.

$$
F_{k, t}=w_{k, t} / \sigma_{k, t}
$$

Using GMM model, the background identification circuit with $\mathrm{K}$ Gaussian distribution of each pixel is checked by match condition and is

$$
M_{k}=1 \text { if } \mid\left(\text { pixel }-\mu_{\mathrm{k}, \mathrm{t}}\right) \mid<\lambda * \sigma_{\mathrm{k}, \mathrm{t}}
$$

Where $\lambda=2.5$, a threshold value.

The equation (2) considered one main part of background identification model and equation (2) verifies with more than one Gaussian distributions and matches with pixel $\mathrm{Mk}=1$

and the Fitness $\left(F_{k, t}\right)$ highest value acts as a "Matched distribution" and its parameters are updated according to the statistical model as below.

$$
\begin{aligned}
& \mu_{k, t}+1=\mu_{k, t}+\alpha_{k, t} \cdot\left(\text { pixel }-\mu_{k, t}\right) \\
& \sigma_{k, t}^{2}+1=\sigma_{k, t}^{2}+\alpha_{k, t} \cdot\left[\left(\text { pixel }-\mu_{\mathrm{k}, t}\right)^{2}-\sigma_{\mathrm{k}, \mathrm{t}}^{2}\right] \\
& w_{k, t}+1=w_{k, t}-\alpha w \cdot w_{k, t}+\alpha w--- \\
& \text { matchsum }_{k, t}+1=\text { matchsum }_{k, t}+1 .
\end{aligned}
$$

If Gaussian distributions are unmatched, mean and variance are remains same and weights are updated as below

$$
w_{k, t}+1=w_{k, t}-\alpha_{w} \cdot w_{k, t} .
$$

If "No match condition" is executed, means the pixel doesn"t match with any Gaussian parameters and smallest value of Fitness $\left(F_{k, t}\right)$ is updated with Gaussian distribution as below

$$
\begin{aligned}
& \mu_{k, t}+1=\text { pixel matchsum } \\
& k, t \\
& \sigma_{k, t}^{2}+1=\text { vinit } w_{k, t}+1=1 / \text { msumtot }
\end{aligned}
$$

Where vinit is initialization value and it is fixed and msumtot is values of total sum of match sum of highest Fitness $\left(F_{k, t}\right)$ with K-1 Gaussian distribution.

\section{Background Identification Model :}

\section{Volume 5 Issue 3, March 2016}




\section{International Journal of Science and Research (IJSR) \\ ISSN (Online): 2319-7064 \\ Index Copernicus Value (2013): 6.14 | Impact Factor (2014): 5.611}

The background identification model operated by the below algorithm as follows.

$$
B=\arg \min _{b}\left(\sum_{k=1}^{b} w_{k, t}>T\right)
$$

The initial b Gaussian distribution are added with weights form (6) and it is sorted from one of the value with highest value of $\left(F_{k, t}\right)$ and its sum is greater than the fixed threshold value , $T^{\mathrm{e}}$ and interval between $[0,1]$. The verified Gaussian distribution is set as Background identification $\mathrm{Bg}$ and if the pixel matches with one the Gaussian distribution parameters then the pixel is Bg pixel and if pixel is not matched with "No match" condition

Then the pixel is Fg.

The conceptual hardware architecture of a Background identification system as shown in the figure 1.

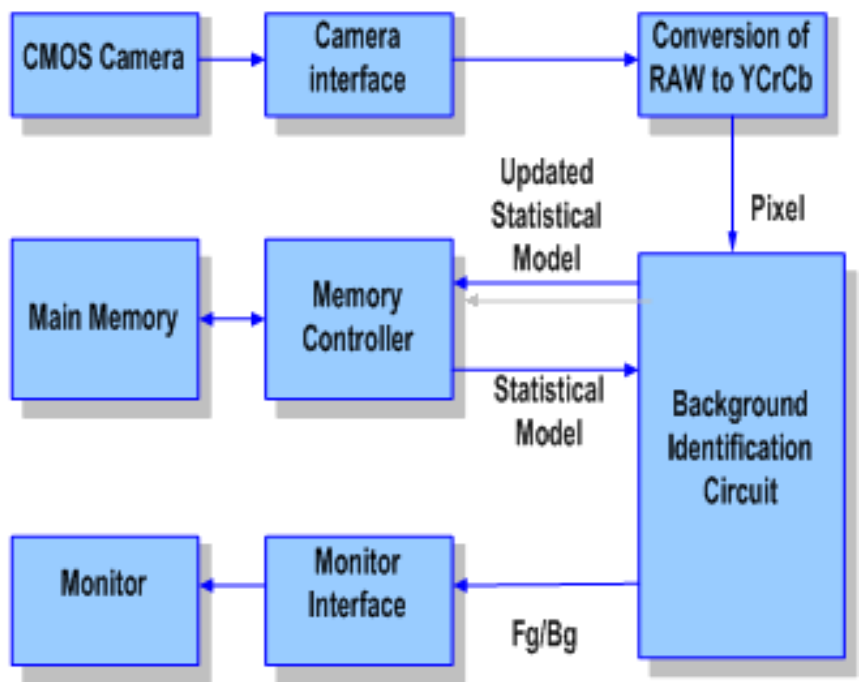

Figure 1: Hardware architecture of a background identification system

The above figure 1 shows the Hardware architecture of a background identification system and it is implemented on FPGA . The vedio sequence is captured by frame by frame using CMOS Camera. The CMOS Interface provides a platform to send pixel values to the background identification circuit and it converts the RAW values to $\mathrm{YCrCb}$ values before given to background identification circuit. The background identification circuit process the both the pixel values of the given frame from $\mathrm{YCrCb}$ values i.e. luminance and "Statistical Model". The background identification circuit gives two different outputs; one is updated statistical model and Fg/Bg. The Gaussian parameters are updated for each pixel and it is read form External memory controller and once a parameter are updated for each pixel is stored in main memory. The $\mathrm{Fg} / \mathrm{Bg}$ provides the output binary image for given input vedio sequence of each frame and it can be displayed on Monitor using monitor interface.

The figure 2 shows the Block diagram of Bg identification circuit on FPGA and written in verilog using Xilinx ISE Tool and simulated using Modelsim tool. we will capture the video sequence using CMOS Camera and the model for video sequence of each pixel is made by mixture of „ $\mathrm{K}^{\mathrm{ce}}$ Gaussian distributions. The statistical model provides the Gaussian parameter includes the weight, mean, variance and match sum. The weights are improved by learning rates and fitness is sorted the pixel order. Based on the control logic matched data

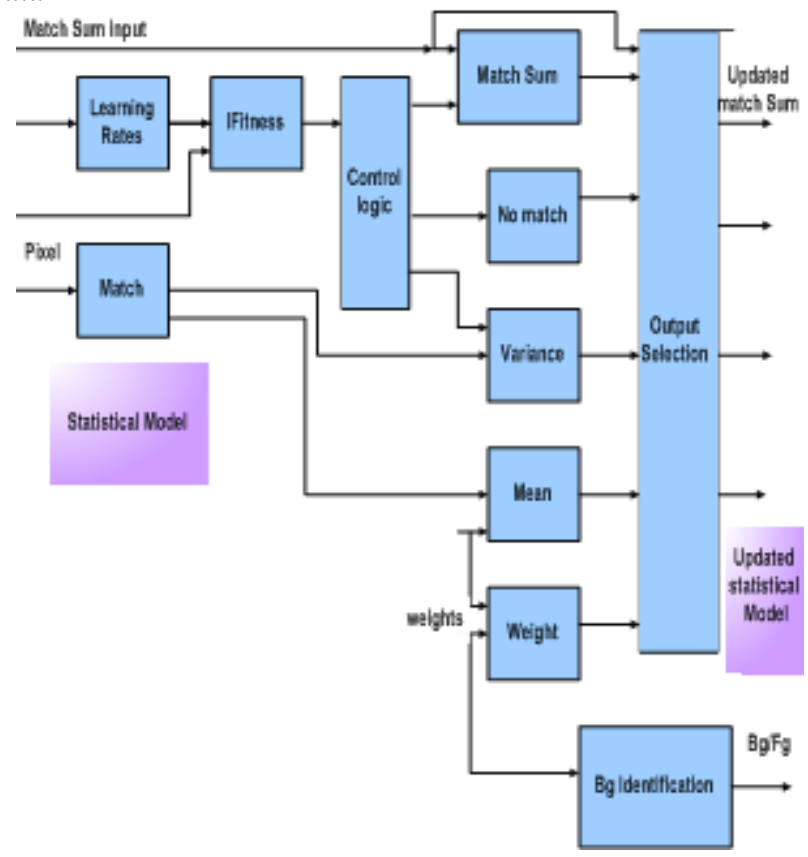

Figure 2: Proposed Bg identification circuit

and No matched data classified. Output selection model provides the updated statistical model output with the help of Bg identification unit. The updated statistical model output are updated in main memory then assigned to final image output.

\section{Conclusion}

We are concluding that The Gaussian mixture model (GMM) algorithm is used to design and implementation of the background identification model on Higher end FPGA . The proposed model works at high speed with real time HD video sequences. We are improving the accuracy with previous design works. The improved area and throughput utilization is achieved with this proposed model. The design model works for lower cost FPGA also. Finally the output image pixel values are analyzed using chip-scope analyzer with Simulated Results.

\section{References}

[1] C. Wren, A. Azarbayejani, T. Darrell, and A. Pentland, "Pfinder: Realtime tracking of the human body," IEEE Trans. Pattern Anal. Mach. Intell., vol. 19, no. 7, pp. 780-785, Jul. 1997.

[2] T. Horprasert, D. Harwood, and L. S. Davis, "A statistical approach for real-time robust background subtraction and shadow detection," in Proc. IEEE Frame-Rate Appl. Workshop, Sep. 1999, pp. 1-19.

[3] C. Stauffer and W. E. L. Grimson, "Adaptive background mixture models for realtime tracking," in Proc. IEEE Comput. Soc. Conf. Comput. Vis. Pattern Recognition., vol. 2 Fort Collins, CO, USA, Jun. 1999, pp. 1-7 


\section{International Journal of Science and Research (IJSR) \\ ISSN (Online): 2319-7064 \\ Index Copernicus Value (2013): 6.14 | Impact Factor (2014): 5.611}

[4] J. Toyama, J. Krumm, B. Brumitt, and B. Meyers, "Wallflower: Principles and practice of background maintenance," in Proc. Int. Conf. Comput. Vis., vol. 1. Sep. 1999, pp. 255-261..

[5] Shao-Yi Chien, Shyh-Yih Ma, and Liang-Gee Chen, Fellow, IEEE, "Efficient Moving Object Segmentation Algorithm Using Background Registration Technique", vol. 12, no. 7, July 2002.

[6] T. Thongkamwitoon, S. Aramvith, and T. H. Chalidabhongse, "An Adaptive Real-time Background Subtraction and Moving Shadows Detection", IEEE International Conference on Multimedia and Expo (ICME), 2004.

[7] Julio Cezar Silveira Jacques Jr Cl'audio Rosito Jung , "Background Subtraction and Shadow Detection in Grayscale Video Sequences", CROMOS Laboratory PIPCA,briliz, XVIII Brazilian Symposium on Computer Graphics and Image Processing ,SIBGRAPI ${ }^{\mathrm{e}}-2005$.

[8] S. Minghua, A. Bermak, S. Chandrasekaran, and A. Amira, "An efficient FPGA implementation of Gaussian mixture models-based classifier using distributed arithmetic," in Proc. 13th IEEE Int. Conf. Electron. Circuits Syst., Dec. 2006, pp. 1276-1279.

[9] Z. Chaohui, D. Xiaohui, X. Shuoyu, S. Zheng, and L. Min, "An improved moving object detection algorithm based on frame difference and edge detection," in Proc. 4th Int. Conf. Image Graph., Aug. 2007, pp. 519-523.

[10]Daming Zhang1, 2, Hui Guo3, Bin Luo1 "an algorithm for estimating number of components of Gaussian mixture model based on penalized distance", China, June 8 10, 2008.

[11]Peng Suo, Yanjiang Wang, "An Improved Adaptive Background Modeling Algorithm Based on Gaussian Mixture Model", P.R.China, 2008.

[12] S. Y. Elhabian, K. M. El-Sayed, and H. A. Sumaya, "Moving object detection in spatial domain using background removal techniquesstate- of-art," in Proc. Recent Patents Comput. Sci., vol. 1. Jan. 2008, pp. 3234.

[13] Yan Li, Lei Li, "A Novel Split and Merge EM Algorithm For Gaussian Mixture Model”, 2009 IEEE.

[14] GAO Ming-ming,Chang Tai-hua, GAO Xiang-xiang "Application of Gaussian Mixture Model Genetic Algorithm in Data Stream Clustering Analysis", Beijing ,China, 2010.

[15] M. Genovese, E. Napoli, and N. Petra, "OpenCV compatible real time processor for background foreground identification," in Proc. Int. Conf. Microelectron., Dec. 2010, pp. 467-470.

[16] Pushkar Gorur, Bharadwaj Amrutur, "Speeded up Gaussian Mixture Model Algorithm for Background Subtraction", 8th IEEE International Conference on Advanced Video and Signal-Based Surveillance, 2011.

[17] Xuejiao Li, Xiaojun Jing "FPGA Based Mixture Gaussian Background Modeling And Motion Detection", Seventh International Conference on Natural Computation, 2011.

[18] Fan Zhang, Lei Yang, Guangwen Zhang, “Adaptive Fast Gaussian Background Subtraction Algorithm", 2nd International Conference on Computer Science and Network Technology, Beijing, China, 2012.
[19]Ce Guo \#_1, Haohuan Fu _2, Wayne Luk, A FullyPipelined Expectation-Maximization Engine for Gaussian Mixture Models, United Kingdom,2012.

[20] Dr. Eyad I. Abbas, Alaa Abdulhussain Refeis, "Isolated Uttered Words Recognition Based on GMM/HMM Algorithms Using SoPC/Nios II Processor Build on Altera Cyclone II FPGA Chip", The First National Conference for Engineering Sciences FNCES 12, Baghdad, Iraq, 2012.

[21] Mariangela Genovese, Ettore Napoli, “An FPGA-based real-time background identification circuit for 1080p video", Eighth International Conference on Signal Image Technology and Internet Based Systems, 2012.

[22] Jingwen Wu, Hani Hamdan, "Model choice for binnedEM algorithms of fourteen parsimonious Gaussian mixture models by BIC and ICL criteria", IEEE International Conference on System Science and Engineering , Budapest, Hungary, ICSSE 2013.

[23] Mariangela Genovese and Ettore Napoli, "ASIC and FPGA Implementation of the Gaussian Mixture Model Algorithm for Real-Time Segmentation of High Definition video", IEEE transactions on very large scale integration (VLSI) systems, 2014. 